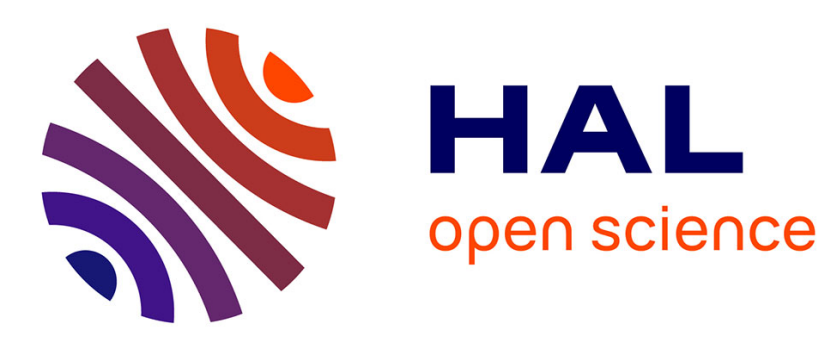

\title{
LoRaWAN Relaying: Push the Cell Boundaries
}

Edouard Lumet, Antonin Le Floch, Rahim Kacimi, Mathieu Lihoreau, André-Luc Beylot

\section{To cite this version:}

Edouard Lumet, Antonin Le Floch, Rahim Kacimi, Mathieu Lihoreau, André-Luc Beylot. LoRaWAN Relaying: Push the Cell Boundaries. 24th ACM International Conference on Modeling, Analysis and Simulation of Wireless and Mobile Systems, Nov 2021, Alicante, Spain. pp.217-220, 10.1145/3479239.3485718. hal-03438762

\section{HAL Id: hal-03438762 https://hal.science/hal-03438762}

Submitted on 22 Nov 2021

HAL is a multi-disciplinary open access archive for the deposit and dissemination of scientific research documents, whether they are published or not. The documents may come from teaching and research institutions in France or abroad, or from public or private research centers.
L'archive ouverte pluridisciplinaire HAL, est destinée au dépôt et à la diffusion de documents scientifiques de niveau recherche, publiés ou non, émanant des établissements d'enseignement et de recherche français ou étrangers, des laboratoires publics ou privés. 


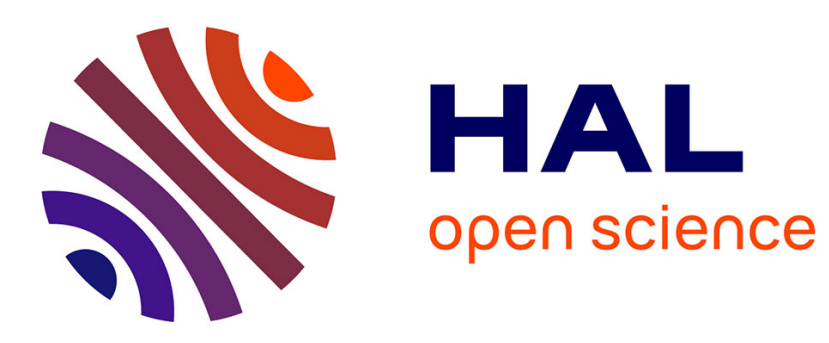

\section{LoRaWAN Relaying: Push the Cell Boundaries}

Lihoreau Mathieu, Edouard Lumet, Antonin Le Floch, Rahim Kacimi, Mathieu Lihoreau, André-Luc Beylot

\section{To cite this version:}

Lihoreau Mathieu, Edouard Lumet, Antonin Le Floch, Rahim Kacimi, Mathieu Lihoreau, et al.. LoRaWAN Relaying: Push the Cell Boundaries. MSWiM '21: 24th ACM International Conference on Modeling, Analysis and Simulation of Wireless and Mobile Systems, Nov 2021, Alicante Spain, France. pp.217-220, 10.1145/3479239.3485718 . hal-03438762

\section{HAL Id: hal-03438762 \\ https://hal.archives-ouvertes.fr/hal-03438762}

Submitted on 22 Nov 2021

HAL is a multi-disciplinary open access archive for the deposit and dissemination of scientific research documents, whether they are published or not. The documents may come from teaching and research institutions in France or abroad, or from public or private research centers.
L'archive ouverte pluridisciplinaire HAL, est destinée au dépôt et à la diffusion de documents scientifiques de niveau recherche, publiés ou non, émanant des établissements d'enseignement et de recherche français ou étrangers, des laboratoires publics ou privés. 


\section{LoRaWAN Relaying: Push the Cell Boundaries}

\author{
Edouard Lumet \\ edouard.lumet@irit.fr \\ University of Toulouse \\ IRIT-ENSEEIHT \\ Toulouse, France
}

\author{
Antonin Le Floch \\ antonin.lefloch@irit.fr \\ University of Toulouse \\ IRIT-ENSEEIHT \\ Toulouse, France
}

\author{
Rahim Kacimi \\ rahim.kacimi@irit.fr \\ University of Toulouse \\ IRIT-UT3 \\ Toulouse, France
}

\author{
Mathieu Lihoreau \\ mathieu.lihoreau@univ-tlse3.fr \\ Research Center on Animal Cognition \\ (CRCA) \\ Center for Integrative Biology (CBI) \\ CNRS, University of Toulouse \\ Toulouse, France
}

\author{
André-Luc Beylot \\ andre-luc.beylot@enseeiht.fr \\ University of Toulouse \\ IRIT-ENSEEIHT \\ Toulouse, France
}

\begin{abstract}
Although LoRa modulation is known for its robustness allowing devices to communicate kilometers away, it suffers from coverage issues especially where density of gateways is low or in dense urban areas. However, a simple 2-hop LoRaWAN communication can seamlessly extend the network coverage and even improve both data extraction rate (DER) and energy consumption. Experiments in this paper figure out cases under non line of sight (NLoS) conditions where relaying performs better. Regarding the exponential increase of airtime with the spreading factor (SF), as soon as a 2-hop SF7 link allows a better DER as a single hop SF8 link, it becomes more attractive to use a relay. Indeed, energy consumption is linked to the airtime - or the amount of time to send a frame - explaining the energy efficiency with the 2-hop relaying protocol. To verify this assert, LoRa network coverage with a testbed in urban environmens is first compared. Then, simulations help to study energy consumption according to the case study. Results prove that relaying effectively gives better results under NLoS conditions, particularly in dense areas, by improving the DER. It also highlights the limits of LoRa in urban areas where the DER can be under 0.5 using SF12 and with less than a kilometer range.
\end{abstract}

\section{CCS CONCEPTS}

- Networks $\rightarrow$ Network experimentation.

\section{KEYWORDS}

LoRaWAN; Relaying; Experiments; Data Extraction Rate; EnergyEfficiency
ACM Reference Format:

Edouard Lumet, Antonin Le Floch, Rahim Kacimi, Mathieu Lihoreau, and AndréLuc Beylot. 2021. LoRaWAN Relaying: Push the Cell Boundaries. In Proceedings of the 24th ACM International Conference on Modeling, Analysis and Simulation of Wireless and Mobile Systems (MSWiM '21), November 22-26, 2021, Alicante, Spain. ACM, New York, NY, USA, 4 pages. https: //doi.org/10.1145/3479239.3485718

\section{INTRODUCTION}

In many environments, a LoRa-enabled gateway allows a network coverage up to a few kilometers using the good parameters regarding the throughput. In fact, it seems to be enough to cover a field or a district town but this idea is often based on a log-distance path model [9]. As discussed in [5], experiments presented in this paper actually show that this model is not accurate in some realistic cases, in particular in rough environments such as dense urban areas which suffer from shadowing effects due to many obstacles. Under these conditions, solutions have to be found in order to extend the LoRa network coverage. Increasing the spreading factor (SF) could be considered, however it leads to higher power consumption and, as shown in experiments presented in this paper, it may be not sufficient. Afterward, a common solution is to deploy more gateways to extend the coverage of low SF and the global coverage [4]. Alternatively, the nodes can be used as relays which seems as simpler and cost-effective implementation while this does not require neither network backhauling nor power line [4].

The presented work aims to tackle an issue observed in constrained environments where the data extraction rate (DER) - which is like a packet delivery ratio (PDR) in other networks - could be much lower than expected even within a short range. Relaying in LoRa seems to be a promising solution to improve the link quality [6], however the tested environments and use cases are quite limited. Indeed in [7] and [8], energy consumption is not considered whereas it is a key challenge in low-power wide area networks (LPWAN). Similarly in [2] authors presented an experiment with a power supplied relay node. Our comprehensive study demonstrates how relaying can efficiently avoid obstacles and connect isolated nodes while saving energy and without generating an overhead in terms of implementation complexity. Relaying in urban environment is experimented and a comparison of the network 
coverage with and without the presence of a relay is given. Finally, results show an increase of both DER and coverage together with an improvement of the energy consumption. Indeed, let us note that airtime (transmission time) approximately doubles with SF resulting on a higher energy consumption and a lower DER at a constant amount of time.

At last, the contributions of this paper can be summarized as: running an experimental campaign including two scenarios in a urban environment; bringing the demonstration of the relevance of relaying to improve DER, network coverage and energy consumption in common real-life case.

The rest of the paper is organized as follows. Section 2 presents a testbed setup and experiments guidelines. Section 3 shows results and analyse and section 4 concludes the paper and draws some directions for the future work.

\section{EXPERIMENTAL DESIGN}

To figure out cases where LoRa relaying seamlessly improves either the network coverage or the DER compared to single hop communications, we set up experiments in two steps. First, we studied the network coverage of a classical single hop LoRa network using SF7 and spotted a place for the relay node given the field properties. Second, we added the relay node and studied again the network coverage in particular in locations where the DER is low or in not covered locations by the gateway. When using a relay, we assumed using only SF7 as we aim to improve both energy consumption and DER.

To get an accurate comparison between single hop and 2-hop links, we also measured the DER using SF8 and SF12. By measuring the DER with SF8, we know if relaying is more effective as a single hop link with an equivalent energy consumption. SF12 is an interesting benchmark as it should allow the maximum range because of its robustness. Thus, this analytical approach is applied in a constrained environment (see 2.1) to compare the performance of relaying in terms of reliability, network range and energy consumption.

\subsection{Case studies}

In order to test how our relaying protocol behaves, we selected a city center as a constrained area. The location of the urban area is shown in figure 1 . The area is globally dense with small buildings and narrow streets. We believed this location would be interesting to pointing out shadowing effect and the benefit of street canyon for the relay location. Indeed, long, straight and narrow streets, known for a beneficial signal propagation, may help extend the network coverage but are also subject to shadowing. For this reason, we first placed the relay node in location $P 7$ which was the entrance of a main straight street allowing LoS or alike conditions. As a second scenario, we then considered location $P 8$ as DER with SF7 was higher and because the presence of two squares let us expect a field open enough for relaying.

\subsection{Testbed setup}

To carry out the experimental design, we set up a simple testbed composed of: (i) a Mikrotik wAP LoRa8 kit with a $6.5 \mathrm{dBi}$ omnidirectionnal outdoor antenna; (ii) a Raspberry Pi 3 B+; (iii) two Pycom expansion boards equipped with LoPy4 modules.

On the Raspberry Pi, we installed Chirpstack network and application servers and an InfluxDB time series database to record frames generated during the experiment. Data visualization was enabled by Grafana for a live watch of the received packets.

Programming language for Pycom boards was MicroPython, a lightweight Python with embedded libraries for microcontrollers [10]. The main advantage of MicroPython is the low memory it consumes, however, as it embeds few libraries, the programming possibilities are limited. For this reason, the experiments were based on the LoRa-LoRaWAN relaying protocol as the function to decrypt LoRaWAN frames is not available in those Pycom boards. LoPy4 modules support Wi-Fi, Bluetooth Low Energy (BLE), Sigfox and LoRa (EU433, EU868 and US915). Given our location, we selected the EU868 frequency plan.

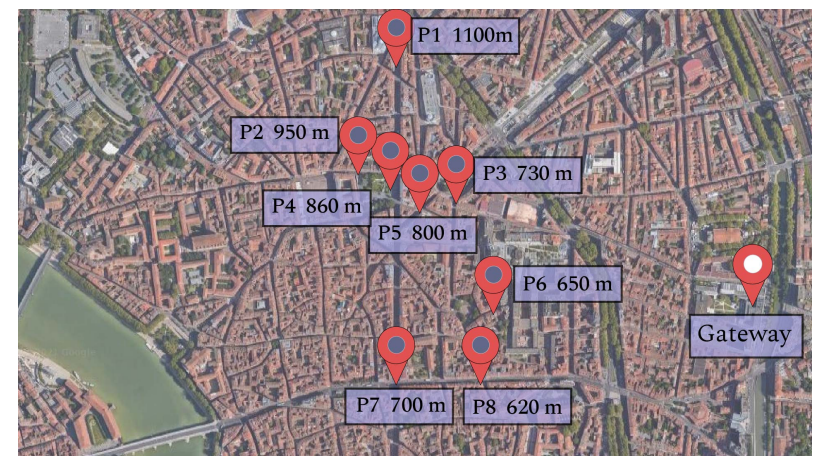

Figure 1: City center of Toulouse, IRIT-ENSEEIHT, representative of dense urban environment.

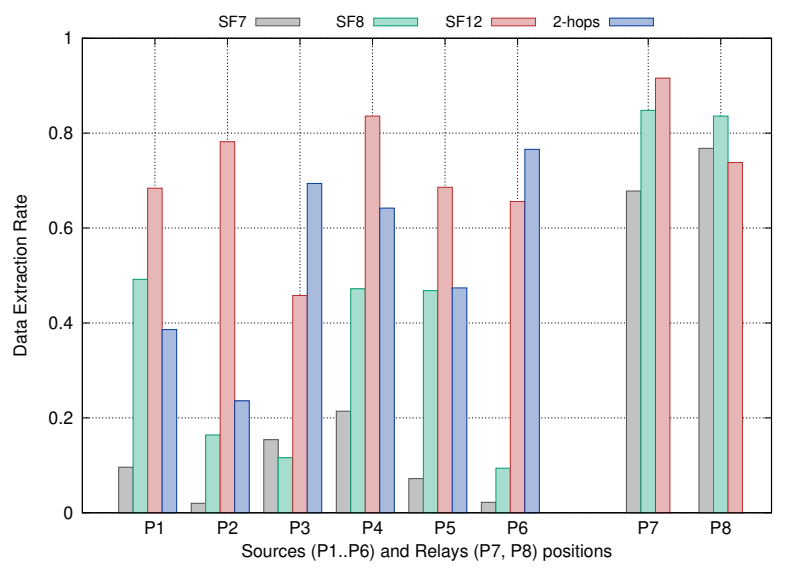

Figure 2: DER comparison between single hop communications and relaying. Relays are located at $P 7$ and $P 8 . P 8$ relays $P 6$ and $P 3$ while $P 7$ relays the others. 


\begin{tabular}{|c|c|c|}
\hline SF & SNR & Sensitivity \\
\hline 7 & -7.5 & -126.5 \\
8 & -10 & -127.25 \\
9 & -12.5 & -131.25 \\
10 & -15 & -132.75 \\
11 & -17.5 & -133.25 \\
12 & -20 & -134.5 \\
\hline
\end{tabular}

Table 1: LoRa SNR and sensitivity thresholds for $125 \mathrm{kHz}$ bandwidth in $\mathrm{dB}$.

\section{RESULTS AND DISCUSSION}

\subsection{Reliability}

As the DER is the ratio of received frames to the number of sent frames, it can be considered as a metric to monitor the link quality. It is also linked to energy consumption because of the retransmissions needed for each lost frame. We also could have considered additional metrics such as the received signal strength indicator (RSSI) and the signal-to-noise ratio (SNR) but these are less relevant. As we observed in the motivating example, a communication can be characterized by a low DER with both RSSI and SNR beyond the common thresholds given in table 1. Moreover, when RSSI and SNR are below the thresholds either the system cannot receive the frame or cannot decode it. In both cases, it causes a lost frame hence a lower computed DER.

Figure 2 shows a poor global link quality considering the first seven measurement sites. The maximum DER reached with SF7 for $P 1$ to $P 7$ is equal to 0.214 while most of them are under 0.1 . This first observation suggested we could expect low DER with SF8 and a real benefit using a relay node. Looking at both single hop SF8 and 2-hop SF7 DER for each site, shows a significant improvement of the DER in $P 2, P 3, P 4$ and $P 6$ while relaying with a value up to 8 times better than single hop SF8 communication. $P 6$ is a relevant example of relaying performance which may be explained by the field properties. Indeed, this measurement site was located in an open square, however it was surrounded by buildings so any connected device, like for instance smart bins, would be affected in the same way as the sensor device. Hence a significant improvement while using a relay node as we placed it at the junction of the main street and a narrow street as discussed in 2.1. $P 3$ gives a similar result. At $P 1$, the distance between the end-node and the relay node is quite important considering low gain antennas. For this reason, the DER is lower with relaying than with single hop SF8.

\subsection{Energy consumption}

The DER is linked to energy consumption as each lost frame should be retransmitted later. The retransmission mechanism can be implemented either at the LoRaWAN level or at the application layer. Here we considered a retransmission for each lost frame and we compared energy consumption by computing airtime of transmissions and restransmissions, according to the SF and the DER. Table 2 shows various airtimes according to the SF. Indeed, airtime depends on the combination of payload size, SF, bandwidth and coding rate. Thus we also considered a fixed payload size, a fixed bandwidth and a fixed coding rate allowing to compute airtime according to the SF only. Finally, energy consumption of a device is linked to the amount of time with radio activity. This is why we chose of airtime as the metric, besides the DER, for power consumption.

Here, we aimed to determine if a 2-hop SF7 communication would consume less than a single hop communication with an equivalent $\mathrm{SF}$ in terms of airtime and DER.

\begin{tabular}{|c|c|c|}
\hline SF & LoRa airtime & LoRaWAN airtime \\
\hline 7 & 29 & 52 \\
8 & 58 & 93 \\
9 & 116 & 186 \\
10 & 232 & 330 \\
11 & 463 & 660 \\
12 & 926 & 1319 \\
\hline
\end{tabular}

Table 2: Measured LoRa and LoRaWAN airtime in $\mathrm{ms}$ according to $\mathbf{S F}$ with $\mathbf{B W}=125 \mathrm{kHz}, \mathbf{C R}=4 / 8,1-$ byte payload

To show how energy efficient is the proposed solution, we computed how much energy is consumed by every node in the benchmark network. As it was not possible for us to precisely measure the LoPy's voltage, we used electrical characteristics given by the LoPy datasheet (table 3) and the simulator LoRaSim [1]. In this approach, we first generated the same topology as in the testbed. We then simulated emissions of 500.000 frames in order to have little confidence intervals.

Every message had a probability to be correctly transmitted according to the node's DER. If it did not succeed, the node tried to retransmit it. When relaying, both node and relay DER were taken into account. We also took into account the energy used by the relay for listening and sending the data. Adding up every transmission, retransmissions and relaying costs gave us the total energy spent to communicate.

\begin{tabular}{|c|c|}
\hline LoPy Mode & Intensity in Ampere \\
\hline Supply current in transmitter mode & $20 \mathrm{~mA}$ \\
Supply current in receiver & $11.5 \mathrm{~mA}$ \\
\hline
\end{tabular}

Table 3: "LoRa electrical characteristics" from LoPy4 Datasheet V1.1 page 19 [11].

Figure 3 plots a communication at different SF. It also shows the total energy consumed when relaying. The selected node is based on a perfect example where relaying drastically improved DER. When so - as shown by the simulations - the energy saving is impressive. Indeed, relaying consumed, respectively, a half and $34 \%$ of the energy consumed using, respectively, SF7 and SF8. 


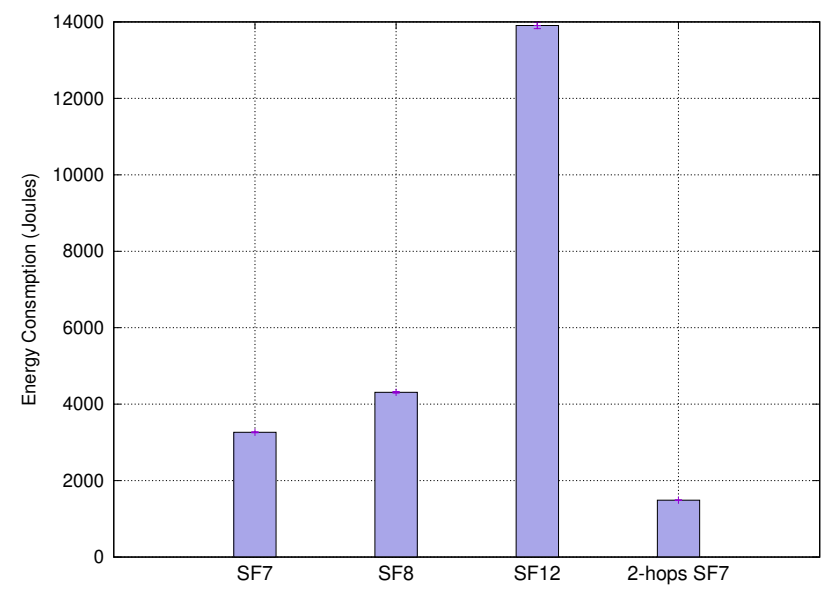

Figure 3: Energy consumed by the node at P6 (cf. fig. 1) in Joules.

\subsection{Conclusive remarks}

To summarize, by considering the significant improvement of the DER while relaying compared to single hop SF8 as shown in 3.2, we demonstrated the relevance of a 2-hops relaying protocol. Indeed, in urban environment the DER is improved within five cases out of six. More importantly, relaying outperforms an SF12 single hop link which is a significant improvement as it has the worst energy consumption.

It is also worth noting that, the use of lowest spreading factors significantly enhances the channel utilization as the airtime tremendously varies between SF7 and SF12 (as shown in fig. 2). In other words, there is a linear relation between energy consumption and channel utilization as both are based on the airtime. Thus, the presented ratios in section 3.2 between the energy consumption of single and 2-hops communications are similar if we consider channel utilization.

Finally, adding new gateways to the network in order to improve both network coverage and reliability requires a power-line and network backhauling which is not practically sustainable. Moreover, it adds complexity in the network with mechanisms as routing, load balancing, and gateway selection [3]. Precisely, under deployment constraints as mentioned above, relaying is undoubtedly relevant as it outperforms even SF12 single hop communication links.

\section{CONCLUSION}

In this paper, we have proposed a relaying protocol which aims to improve the reliability and the energy efficiency of data-collection in LoRaWAN networks. We have introduced and implemented new relaying mechanisms such as synchronization, end-node identity preservation, and buffering which allows to tackle down-link issues by guaranteeing its effectiveness. Through extensive experimental study, we have proved the relevance of relaying to improve the data extraction rate compared to single hop SF8 communication. In addition, we have improved a simulation framework by our experimental measurements in order to evaluate the energy-efficiency of our design in large-scale networks. Undoubtedly, relaying in
LoRa networks can significantly improve the link quality and the reliability while maximizing the network lifetime.

This work is not the last chapter on relaying in LoRaWAN. Indeed, there is room to extend the performance evaluation before a potential democratization of relaying over private and operated LoRaWAN networks. This extension spans across multiple dimensions, including, but not limited to, testing in more diverse environment densities, testing a balanced selection of relay nodes, proving how avoiding SF12 one-hop communication can significantly reduce interference and collisions by relaying through low Time-On-Air SF7 communications.

\section{ACKNOWLEDGMENTS}

This work has been supported by the 'SASHA' project funded by Federal University of Toulouse and the Occitania Regional Fund. It is also partially supported by the neOCampus research \& innovation operation of Toulouse University.

\section{REFERENCES}

[1] Martin C. Bor, Utz Roedig, Thiemo Voigt, and Juan M. Alonso. 2016. Do LoRa LowPower Wide-Area Networks Scale?. In Proceedings of the 19th ACM International Conference on Modeling, Analysis and Simulation of Wireless and Mobile Systems (MSWiM '16). Association for Computing Machinery, New York, NY, USA, 59-67. https://doi.org/10.1145/2988287.2989163

[2] S. S. Borkotoky, U. Schilcher, and C. Bettstetter. 2019. Cooperative Relaying in LoRa Sensor Networks. In 2019 IEEE Global Communications Conference (GLOBECOM). 1-5. https://doi.org/10.1109/GLOBECOM38437.2019.9014071 ISSN: 25766813.

[3] Roger Pueyo Centelles, Felix Freitag, Roc Meseguer, and Leandro Navarro. 2021. Beyond the Star of Stars: An Introduction to Multihop and Mesh for LoRa and LoRaWAN. IEEE Pervasive Computing 20, 2 (April 2021), 63-72. https://doi.org/ 10.1109/MPRV.2021.3063443 Conference Name: IEEE Pervasive Computing.

[4] Jeferson Rodrigues Cotrim and João Henrique Kleinschmidt. 2020. LoRaWAN Mesh Networks: A Review and Classification of Multihop Communication. Sensors 20, 15 (Jan. 2020), 4273. https://doi.org/10.3390/s20154273 Number: 15 Publisher: Multidisciplinary Digital Publishing Institute.

[5] Ousmane Dieng, Congduc Pham, and Ousmane Thiare. 2020. Comparing and Adapting Propagation Models for LoRa Networks. In 2020 16th International Conference on Wireless and Mobile Computing, Networking and Communications (WiMob). 1-7. https://doi.org/10.1109/WiMob50308.2020.9253410 ISSN: 21604894.

[6] M. DIOP and C. PHAM. 2019. Increased flexibility in long-range IoT deployments with transparent and light-weight 2-hop LoRa approach. In 2019 Wireless Days (WD). 1-6. https://doi.org/10.1109/WD.2019.8734228 ISSN: 2156-972X.

[7] Olivier Flauzac, Joffrey Herard, Florent Nolot, and Philippe Cola. 2020. A Low Power LoRa-LoRaWan Relay Function with a Single Input, Single Output Device. In Proceedings of the 2020 International Conference on Embedded Wireless Systems and Networks on Proceedings of the 2020 International Conference on Embedded Wireless Systems and Networks (EWSN'20). Junction Publishing, USA, 283-288.

[8] Olivier Flauzac, Joffrey Hérard, Florent Nolot, and Philippe Cola. 2020. A Fault Tolerant LoRa/LoRaWAN Relay Protocol Using LoRaWAN Class A Devices. In Ad-Hoc, Mobile, and Wireless Networks (Lecture Notes in Computer Science), Luigi Alfredo Grieco, Gennaro Boggia, Giuseppe Piro, Yaser Jararweh, and Claudia Campolo (Eds.). Springer International Publishing, Cham, 295-302. https://doi.org/10.1007/978-3-030-61746-2_22

[9] Pascal Jörke, Stefan Böcker, Florian Liedmann, and Christian Wietfeld. 2017. Urban Channel Models for Smart City IoT- Networks Based on Empirical Measurements of LoRa-links at 433 and $868 \mathrm{MHz}$. IEEE, Montreal, QC, Canada, 6. https://doi.org/10.1109/PIMRC.2017.8292708

[10] MicroPython. 2021. MicroPython programming language official website. Retrieved June 18, 2021 from https://micropython.org/

[11] Pycom. 2020. LoPy4 V2 Datasheet Version 1.1. Retrieved June 15, 2021 from https://docs.pycom.io/gitbook/assets/specsheets/Pycom_002_Specsheets_ LoPy4_v2.pdf 